\title{
First 28: Design of a Mobile App for Neonatal Health Risk Assessment and Support for New Mothers
}

Andy Pham ${ }^{1 *}$, BS; Elsie F Bluett ${ }^{1 *}$, BS; Priyanka Puthran ${ }^{1 *}$, BE, MSc; Sayantani Sarkar ${ }^{2 *}$, BSC; Katherine K Kim ${ }^{2 *}$, MBA, MPH, PhD; Prabhu R Shankar ${ }^{1 *}$, MD, MS

\footnotetext{
${ }^{1}$ Division of Health Informatics, Department of Public Health Sciences, University of California Davis, Sacramento, CA, United States

${ }^{2}$ Betty Irene Moore School of Nursing, University of California Davis, Sacramento, CA, United States

*all authors contributed equally
}

\section{Corresponding Author:}

Andy Pham, BS

Division of Health Informatics

Department of Public Health Sciences

University of California Davis

4610 X Street

Sacramento, CA,

United States

Phone: 7347131

Email: apham@ucdavis.edu

\begin{abstract}
Background: Factors like dehydration and respiratory infection pose risks to infant survival in the critical first 28 days of life. UNICEF reported the 2016 global rate of neonatal death was 19 per 1000 live births. Typically, women manage multiple household and family responsibilities in addition to care of a new baby and often feel overwhelmed by the demands of new motherhood. The American College of Obstetricians and Gynecologists recommends that support to new mothers be an ongoing process, rather than a single postnatal visit. However, in low-resource environments such as developing countries and remote communities, access to ongoing support for breastfeeding, health education, and infant check-ups from a professional health care provider or health worker may not be possible. Numerous examples exist of successful mobile health interventions in low-resource environments. However, existing mobile apps for newborn health often focus on single issues that are disconnected from health care providers. There is a need to comprehensively address multiple newborn health issues, with evidence-based and personalized interventions that support new mothers.
\end{abstract}

Objective: This study aims to design and build a prototype of a mobile app to comprehensively identify early signs and symptoms of common newborn illnesses, access relevant evidence-based health information, and support decision-making with the overall goal of enhancing new mothers' ability to improve newborn health outcomes. The prototype will be used in a future pragmatic trial.

Methods: An interdisciplinary and international team including nursing, medicine, dietary, health informatics, and public health collaborated on this study. First, a literature review was conducted to supplement the team's existing knowledge on common neonatal problems, generate the evidence base for appropriate in-home interventions, and identify best practices in breast feeding. Second, a review of current mobile apps available in neonatal risks was conducted to assess gaps with attention to comprehensiveness of health issues, interface/integration with clinical decision support systems, and application of user-centered design and state of the art design principles and standards.

Results: Our app, First 28, works offline for easy accessibility and displays evidence-based best practices and guidelines, personalized for mothers based on risks. Using a tailored symptoms list and computerized data entry to gather information, the mobile app performs analysis using a decision table algorithm to identify the risks the baby might encounter and suggests best solutions based on the outcomes. Mothers can submit images or crucial information about their baby and track growth through the app's data visualization tools. Data is stored on a FHIR server for integration with health care services and electronic health records. Future plans include automated data and image analytics of the uploaded information to alert health care providers of any abnormalities that may provide critical early evidence for potential neonatal risks and complications. 
Conclusions: First 28 empowers mothers with the knowledge and resources to maintain proper breastfeeding techniques, assess newborn health risks, and improve health outcomes within the crucial first 28 days of life. In the next phase, the prototype will be evaluated by users with a plan to utilize it in a pragmatic trial.

(iproc 2018;4(2):e11740) doi: $\underline{10.2196 / 11740}$

\section{KEYWORDS}

mHealth; neonate; neonatal; clinical decision support (CDS); breastfeeding; neonatal health risk; neonatal complication; dehydration; respiratory infection; mother; postnatal; decision table; FHIR

Edited by T Hale; this is a non-peer-reviewed article. Submitted 31.07.18; accepted 29.08.18; published 17.09.18.

Please cite as:

Pham A, Bluett EF, Puthran P, Sarkar S, Kim KK, Shankar PR

First 28: Design of a Mobile App for Neonatal Health Risk Assessment and Support for New Mothers

iproc 2018;4(2):e11740

URL: http://www.iproc.org/2018/2/e11740/

doi: $10.2196 / 11740$

PMID:

(C)Andy Pham, Elsie F Bluett, Priyanka Puthran, Sayantani Sarkar, Katherine K Kim, Prabhu R Shankar. Originally published in Iproceedings (http://www.iproc.org), 17.09.2018. This is an open-access article distributed under the terms of the Creative Commons Attribution License (https://creativecommons.org/licenses/by/4.0/), which permits unrestricted use, distribution, and reproduction in any medium, provided the original work, first published in Iproceedings, is properly cited. The complete bibliographic information, a link to the original publication on http://www.iproc.org/, as well as this copyright and license information must be included. 